\title{
Bi-allelic mutations in uncoordinated mutant number- 45 myosin chaperone $B$ are a cause for congenital myopathy
}

\author{
Hormos Salimi Dafsari ${ }^{1,2}$, Nur Mehpare Kocaturk ${ }^{1,2}$, Hülya-Sevcan Daimagüler ${ }^{1,2}$, Anna Brunn ${ }^{3}$, Jörg Dötsch ${ }^{1,4}$, \\ Joachim Weis ${ }^{5}$, Martina Deckert ${ }^{3}$ and Sebahattin Cirak ${ }^{1,2,4^{*}}$
}

\begin{abstract}
Congenital myopathies (CM) form a genetically heterogeneous group of disorders characterized by perinatal muscle weakness. Here, we report an 11-year old male offspring of consanguineous parents of Lebanese origin. He presented with proximal weakness including Gower's sign, and skeletal muscle biopsy revealed myopathic changes with core-like structures. Whole exome sequencing of this index patient lead to the discovery of a novel genetically defined CM subtype based on bi-allelic mutations in the uncoordinated mutant number-45 myosin chaperone $B$ (UNC45B) NM_173167:c.2261G > A, p.Arg754GIn. The mutation is conserved in evolution and co-segregates within the pedigree with the phenotype, and located in the myosin binding armadillo repeat domain 3 (ARM3), and has a CADD Score of 35. On a multimeric level, UNC45B aggregates to a chain which serves as an assembly line and functions as a "template" defining the geometry, regularity, and periodicity of myosin arranged into muscle thick filaments. Our discovery is in line with the previously described myopathological phenotypes in C. elegans and in vertebrate mutants and knockdown-models. In conclusion, we here report for the first time a patient with an UNC45B mutation causing a novel genetically defined congenital myopathy disease entity.
\end{abstract}

\section{Text}

Congenital myopathies (CM) form a genetically heterogeneous group of disorders characterized by perinatal muscle weakness $[18,20]$. Here, we report an 11-year old male offspring of consanguineous parents of Lebanese origin. He presented with proximal weakness including Gower's sign, and skeletal muscle biopsy revealed myopathic changes with core-like structures. Genomic investigation of this index patient lead to the discovery of a novel genetically defined CM subtype based on bi-allelic mutations in the uncoordinated mutant number- 45 myosin chaperone B (UNC45B) gene. Regarding medical history, the mother reported reduced fetal movements during pregnancy. After birth, the patient presented as a floppy infant with feeding difficulties, improving after the first year of life. He was able to

\footnotetext{
* Correspondence: sebahattin.cirak@uk-koeln.de

'Department of Pediatrics, Faculty of Medicine and University Hospital Cologne, University of Cologne, 50937 Cologne, Germany

${ }^{2}$ Center for Molecular Medicine (CMMC), Faculty of Medicine and University

Hospital Cologne, University of Cologne, 50937 Cologne, Germany

Full list of author information is available at the end of the article
}

sit and walk independently at 10 and 20 months of age, respectively. Currently, his Gower's time is $>10$ s (Fig. 1b), he is unable to run, shows a Trendelenburg sign (Additional file 1: Figure S1h), he is overweight and has a static disease course. He talks with a nasal voice without chewing or swallowing difficulties. Facial weakness and ophthalmoplegia are absent. While he had a reduced forced vital capacity of $70 \%$, his echocardiogram showed a normal heart function. Serum creatinine kinase levels were not elevated. For further clinical details, please see Additional file 2: Supplementary Material.

At the age of 10 years, left femoral quadriceps muscle biopsy showed myopathic changes, i.e., fiber size variability including hypertrophic and atrophic fibers and central nuclei (Fig. 1c) with core-like lesions mainly in the center of muscle fibers (Fig. 1d). Fiber type distribution was altered as type-2 fibers were virtually absent (Fig. 1d, Additional file 1: Figure S1a). Small neonatal myosin positive fibers indicated regeneration (Additional file 1: Figure S1b). Electron microscopy unraveled numerous core-like alterations of myofibrillary architecture 


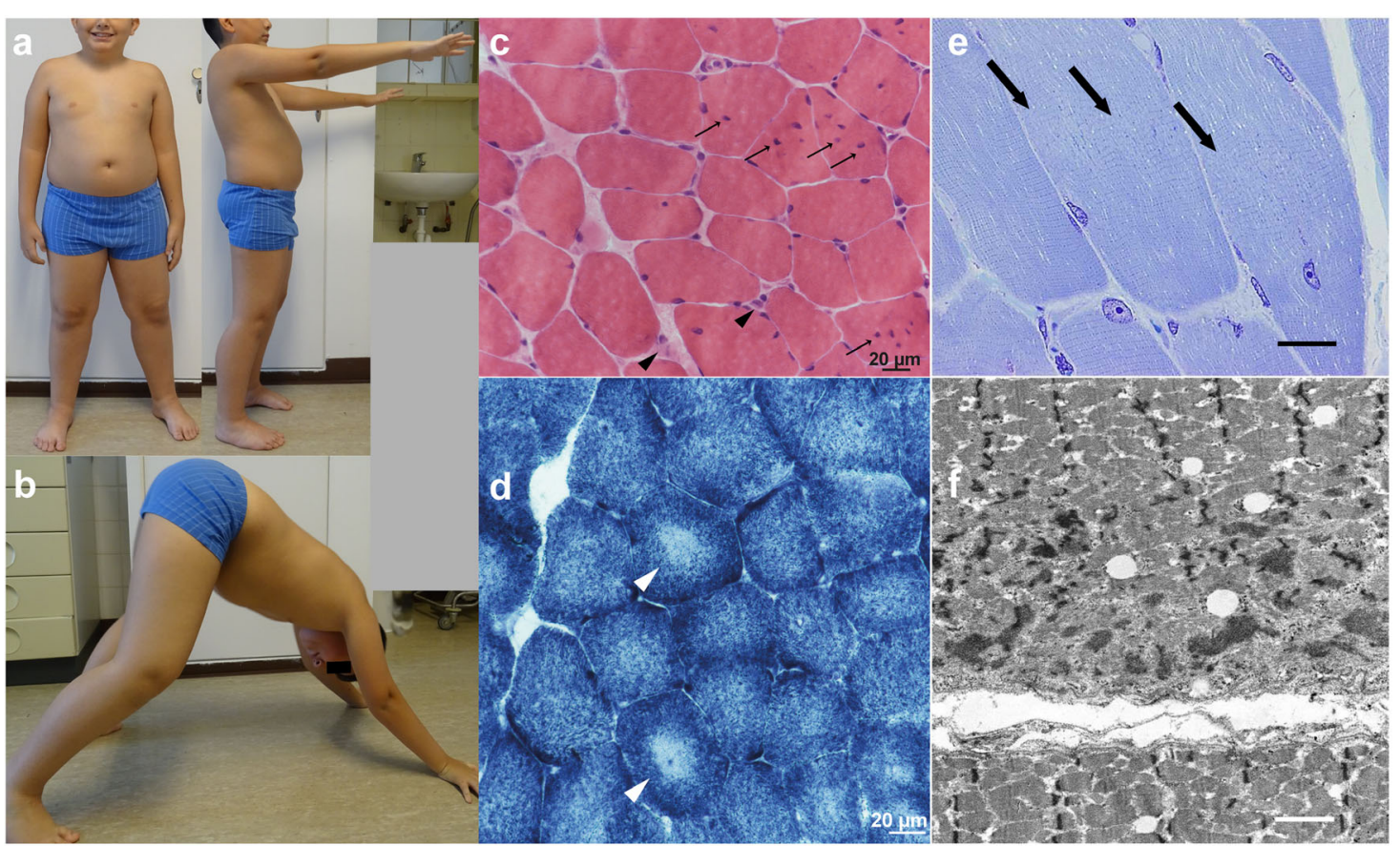

g
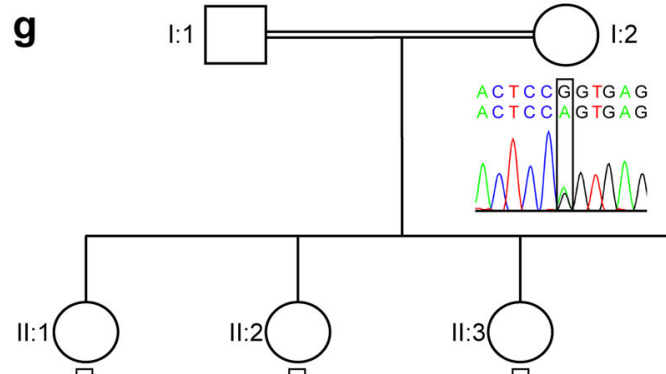

ACTCC GTGA ACTCCGGTGA ACTC NWWWMD

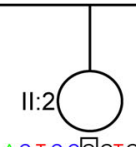

ACTCCGGTGAG ACTC CG GTGAG



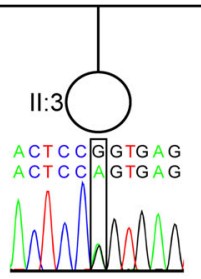
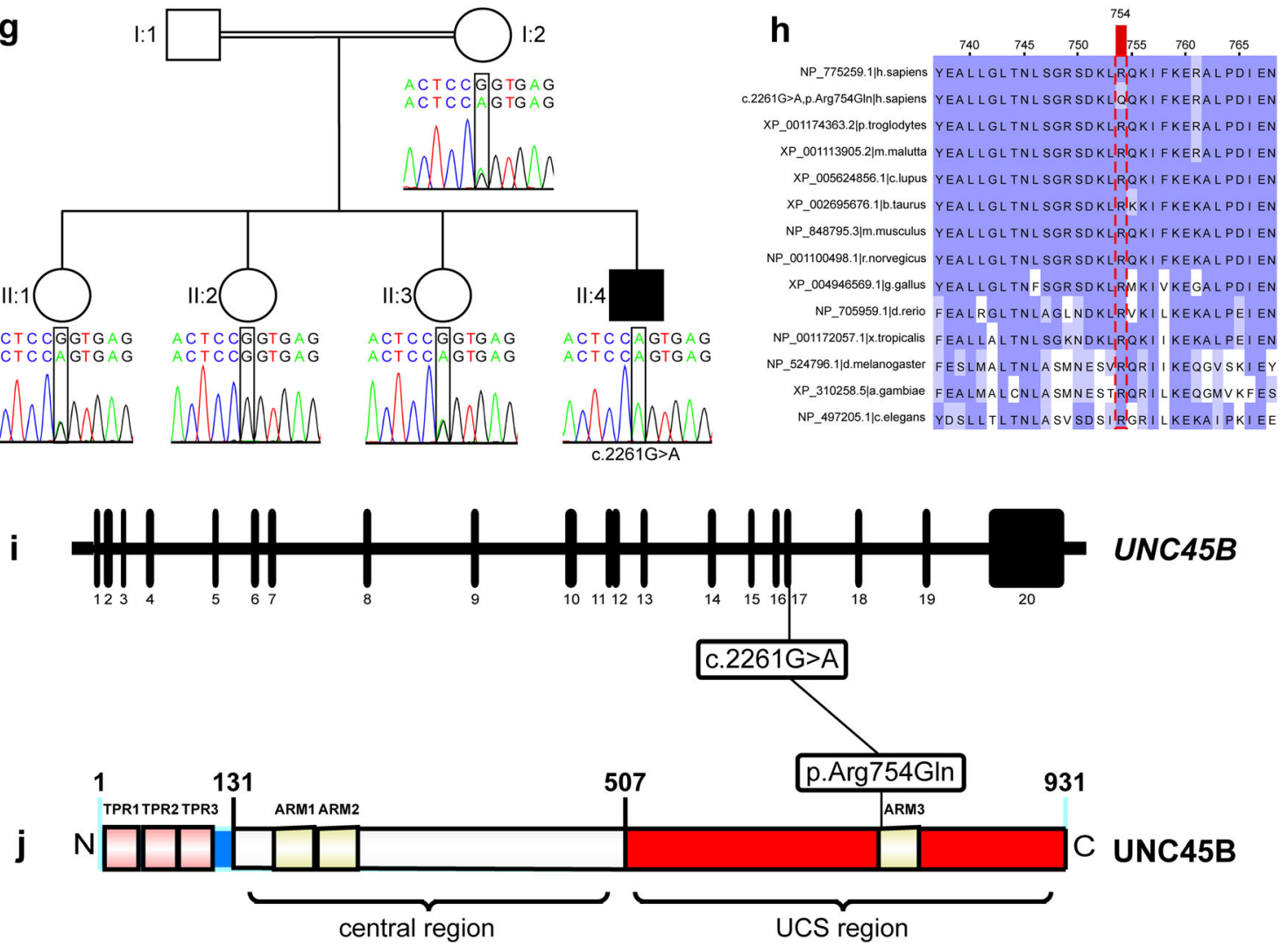

Fig. 1 (See legend on next page.) 


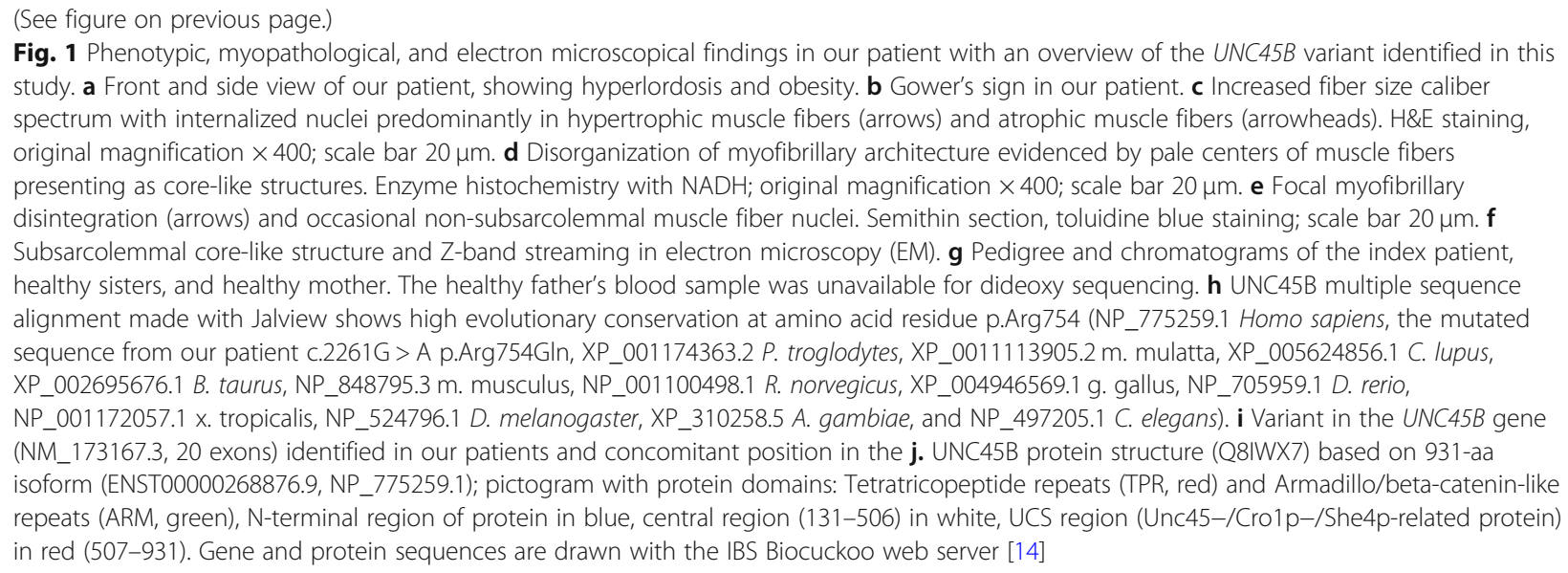

with Z-band streaming (Fig. 1f). Some mitochondria showed prominent matrix granula, globoidal inclusions, and even paracristalline intramitochondrial inclusions (Additional file 1: Figure S1f). We also observed subsarcolemmal accumulations of organelles (Additional file 1: Figure S1e).

To uncover underlying disease-causing mutations, we performed whole-exome sequencing (WES) (Additional file 2: Table S1 and Supplementary Material) [3]. By stringent filtering for various inheritance models (Additional file 2: Table S2), the most likely autosomalrecessive model in a consanguineous family let us to the solution. Based on skeletal muscle expression levels (Additional file 1: Figure S1 g) and previously reported animal models $[4,9,11,13,21]$, we consider a strictly conserved homozygous base pair exchange in UNC45B (NM_173167:c.2261G > A, p.Arg754Gln, Fig. 1g-j) in a homozygous region as pathogenic. The variant leads to an amino acid substitution with a change in polarity and mass (dissimilarity 43 in Sneath's index) in the armadillo repeat domain 3 (ARM3), and is reported with a CADD Score of 35 . UNC45B is highly conserved and constrained against loss-of-function variants in the gnomAD population database (Additional file 2: Table S3). UNC45 proteins show a three-domain configuration, with an $\mathrm{N}$-terminal tetratricopeptide repeat (TPR) domain, poorly conserved central domain, and a C-terminal UCS domain (Unc45-/Cro1p-/She4p-related protein) [13]. Three consensus TPR repeats participate in proteinprotein interaction especially with $\mathrm{Hsp} 70$ and $\mathrm{Hsp} 90$ [17]. The C-terminal UCS domain has been shown to form a putative myosin-binding groove, largely stabilized by electrostatic interactions [6]. Our patient's missense mutation is located in the third ARM domain at residue p.Arg754 at the C-terminal UCS region, which might abrogate the interaction between $\mathrm{UNC} 45 \mathrm{~B}$ and myosin heavy chain [19], thus impairing myofibrillogenesis (Additional file 1: Figure S2d). Indeed, in-silico modeling and docking studies of the human UNC45B protein showed that the binding groove in the UCS domain is a negatively-charged surface at the R18R19 helices of UNC45B and serves as "place-holder" for the charged loop- $\mathrm{U}$ and $\beta$-sheets residues of myosin (MYH7) [6]. The p.Arg754Gln mutation is actually found directly in $\mathrm{R} 18 \mathrm{H} 1[6,12]$. We calculated a change to a decreased isoelectric point and lower net charge from wildtype to p.Arg754Gln mutant in the R18-R19 residues at pH 7.4 by using the Prot-pi tool (Additional file 1: Figure S2c).

Notably, all three isoforms of UNC45B are highly expressed in skeletal muscle and the p.Arg754Gln mutation affects all isoforms (Additional file 1: Figure S2a,b), only one of the three isoforms is highly expressed in cardiac muscle. In a $D$. melanogaster model, an Unc- 45 knockdown showed a severe cardiac phenotype with dilated cardiomyopathy and reduced muscle contractility [15]. Unc-45b knockdown in zebrafish and also the steif/ unc-45b mutants resulted in paralysis and cardiac dysfunction based on severely disrupted myofibrillogenesis $[4,21]$. Therefore, our patient might develop cardiomyopathy at later ages. Knockdown of unc- $45 b$ severely affected sarcomere organization including $\mathrm{M}$ - and Z-lines of skeletal muscles of embryos [2].

From the essential physiological function of UNC45B in muscle development, it can be deducted that deleterious genetic variants may lead to myopathy. On a multimeric level, UNC45B aggregates to a chain which serves as an assembly line for beta ( $\beta$ )-myosin heavy chain, encoded by MYH7 [1, 5], and functions as a "template" defining the geometry, regularity, and periodicity of myosin arranged into muscle thick filaments [16]. After myosin incorporates into thick filaments, Unc45b and Hsp90 dissociate from myosin ensuring the proper myosin filament assembly [15]. Once disassociated, UNC45B binds to a VCP cofactor protein UFD-2 and an E3 ligase CHIP which leads to poly-ubiquitylation of UNC45B and its subsequent proteasomal degradation [10] (Additional file 1: Figure S2d). 
Therefore, we hypothesize that our patient's mutation in UNC45B in the UCS domain might directly lead to myofibrillogenesis failure (Additional file 1: Figure S2d). Of note, a heterozygous missense variant in UNC45B (c.2413C > T, p.Arg805Trp) has been reported to cause a dominant form of glaucoma without further confirmation since publication [8]. Noteworthy, there is a high heterozygous allele carrier status of $18 / 272310$ in gno$\mathrm{mAD}$ of this c.2413C $>\mathrm{T}$ variant in healthy adults.

In conclusion, we here report for the first time a patient with an UNC45B mutation causing a novel genetically defined congenital myopathy disease entity. Our discovery is in line with the previously described myopathological phenotypes in C. elegans and in vertebrate mutants and knockdown-models $[4,7,9,11,19]$.

\section{Supplementary information}

Supplementary information accompanies this paper at https://doi.org/10. 1186/s40478-019-0869-1.

Additional file 1: Figure S1. Further myopathological, electron microscopical and phenotypic findings in our patient with UNC45B variant. Figure S2. Gene and isoform expression of UNC45B in various tissues and a possible disease model scheme.

Additional file 2: Table S1. Detailed metrics of Whole Exome Sequencing in our patient with coverage (1x, 2x, 10x, 20x, 30x, 100x, mean). Table S2. Results of the variant filtering and the specific criteria we applied on the dataset. Table S3. Prediction of pathogenicity for our patient's UNC45B variant via multiple scoring tools.

\section{Acknowledgments}

We furthermore thank the Cologne Center for Genomics (CCG) at the University of Cologne for performing Whole Exome Sequencing of the patient and providing the sequencing data. We also thank the Regional Computing Center of the University of Cologne (RRZK) for providing computing time for the bioinformatics analyses on the DFG-funded HighPerformance Computing (HPC) system CHEOPS.

\section{Authors' contributions \\ Manuscript writing, genetic data and bioinformatic analysis, and sequencing were performed by HSD. NMK contributed to manuscript writing and figure generation. H-SD contributed to the genomic analysis and manuscript writ- ing. Myopathological and electron microscopy examinations were performed and interpreted by $A B, J W$, and MD. Patient recruitment and examination, analysis of WES data, obtainment of funding, study design, manuscript writ- ing, and supervision of the study were contributed by SC. All authors (HSD, NMK, H-SD, AB, JD, JW, MD, SC) have critically reviewed and approved the manuscript.}

\section{Funding}

This work was supported by the Deutsche Forschungsgemeinschaft, Germany grants (Cl 218/1-1) to Dr. Sebahattin Cirak. Dr. Hormos Salimi Dafsari was supported by the Gerok program of the Faculty of Medicine, University of Cologne.

\section{Availability of data and materials}

The next generation sequencing datasets generated and analyzed during the current study are not publicly available because of patient confidentiality and since we do not have informed consent for that.

\section{Ethics approval and consent to participate}

All procedures performed in studies involving human participants were in accordance with the ethical standards of the institutional research committee (ethics committee of the Medical Faculty, University Hospital
Cologne, University of Cologne [17-096]) and with the 1964 Helsinki declaration and its later amendments or comparable ethical standards. Informed consent was obtained from the legal guardian/parent of the index case.

\section{Competing interests}

The authors declare that they have no competing interests.

\section{Author details}

${ }^{1}$ Department of Pediatrics, Faculty of Medicine and University Hospital Cologne, University of Cologne, 50937 Cologne, Germany. ${ }^{2}$ Center for Molecular Medicine (CMMC), Faculty of Medicine and University Hospital Cologne, University of Cologne, 50937 Cologne, Germany. ${ }^{3}$ Institute of Neuropathology, Faculty of Medicine and University Hospital Cologne, University of Cologne, 50931 Cologne, Germany. ${ }^{4}$ Center for Rare Diseases, Faculty of Medicine and University Hospital Cologne, University of Cologne, 50937 Cologne, Germany. ${ }^{5}$ Institute of Neuropathology, RWTH University Hospital, 52074 Aachen, Germany.

Received: 10 December 2019 Accepted: 10 December 2019 Published online: 18 December 2019

\section{References}

1. Ao W, Pilgrim D (2000) Caenorhabditis elegans UNC-45 is a component of muscle thick filaments and colocalizes with myosin heavy chain B, but not myosin heavy chain a. J Cell Biol 148:375-384. https://doi.org/10.1083/jcb.148.2.375

2. Bernick EP, Zhang PJ, Du S (2010) Knockdown and overexpression of Unc$45 \mathrm{~b}$ result in defective myofibril organization in skeletal muscles of zebrafish embryos. BMC Cell Biol 11:70. https://doi.org/10.1186/1471-2121-11-70

3. Dafsari HS, Sprute R, Wunderlich G, Daimaguler HS, Karaca E, Contreras A, Becker K, Schulze-Rhonhof M, Kiening K, Karakulak T, Kloss M, Horn A, Pauls A, Nurnberg P, Altmuller J, Thiele H, Assmann B, Koy A, Cirak S (2019) Novel mutations in KMT2B offer pathophysiological insights into childhood-onset progressive dystonia. J Hum Genet 64:803-813. https://doi.org/10.1038/ s10038-019-0625-1

4. Etard C, Behra M, Fischer N, Hutcheson D, Geisler R, Strahle U (2007) The UCS factor Steif/Unc-45b interacts with the heat shock protein Hsp90a during myofibrillogenesis. Dev Biol 308:133-143. https://doi.org/10.1016/j. ydbio.2007.05.014

5. Etard C, Roostalu U, Strahle U (2008) Shuttling of the chaperones Unc45b and Hsp90a between the a band and the $Z$ line of the myofibril. J Cell Biol 180:1163-1175. https://doi.org/10.1083/jcb.200709128

6. Fratev F, Osk Jonsdottir S, Pajeva I (2013) Structural insight into the UNC-45myosin complex. Proteins 81:1212-1221. https://doi.org/10.1002/prot.24270

7. Geach TJ, Zimmerman LB (2010) Paralysis and delayed Z-disc formation in the Xenopus tropicalis unc45b mutant dicky ticker. BMC Dev Biol 10:75. https://doi.org/10.1186/1471-213x-10-75

8. Hansen L, Comyn S, Mang Y, Lind-Thomsen A, Myhre L, Jean F, Eiberg H, Tommerup N, Rosenberg T, Pilgrim D (2014) The myosin chaperone UNC45B is involved in lens development and autosomal dominant juvenile cataract. Eur J Hum Genet 22:1290-1297. https://doi.org/10. 1038/ejhg.2014.21

9. Hoppe T, Cassata G, Barral JM, Springer W, Hutagalung AH, Epstein HF, Baumeister R (2004) Regulation of the myosin-directed chaperone UNC-45 by a novel E3/E4-multiubiquitylation complex in C. elegans. Cell 118:337349. https://doi.org/10.1016/j.cell.2004.07.014

10. Janiesch PC, Kim J, Mouysset J, Barikbin R, Lochmuller H, Cassata G, Krause S, Hoppe T (2007) The ubiquitin-selective chaperone CDC-48/p97 links myosin assembly to human myopathy. Nat Cell Biol 9:379-390. https://doi. org/10.1038/ncb1554

11. Landsverk ML, Li S, Hutagalung AH, Najafov A, Hoppe T, Barral JM, Epstein HF (2007) The UNC-45 chaperone mediates sarcomere assembly through myosin degradation in Caenorhabditis elegans. J Cell Biol 177:205-210. https://doi.org/10.1083/jcb.200607084

12. Lee CF, Hauenstein AV, Fleming JK, Gasper WC, Engelke V, Sankaran B, Bernstein SI, Huxford T (2011) X-ray crystal structure of the UCS domaincontaining UNC-45 myosin chaperone from Drosophila melanogaster. Structure 19:397-408. https://doi.org/10.1016/j.str.2011.01.002

13. Lee CF, Melkani GC, Bernstein SI (2014) The UNC-45 myosin chaperone: from worms to flies to vertebrates. Int Rev Cell Mol Biol 313:103-144. https://doi.org/10.1016/b978-0-12-800177-6.00004-9 
14. Liu W, Xie Y, Ma J, Luo X, Nie P, Zuo Z, Lahrmann U, Zhao Q, Zheng Y, Zhao $Y$, Xue Y, Ren J (2015) IBS: an illustrator for the presentation and visualization of biological sequences. Bioinformatics (Oxford, England) 31: 3359-3361. https://doi.org/10.1093/bioinformatics/btv362

15. Melkani GC, Bodmer R, Ocorr K, Bernstein SI (2011) The UNC-45 chaperone is critical for establishing myosin-based myofibrillar organization and cardiac contractility in the drosophila heart model. PLoS One 6:e22579. https://doi. org/10.1371/journal.pone.0022579

16. Pokrzywa W, Hoppe T (2013) Chaperoning myosin assembly in muscle formation and aging. Worm 2:e25644-e25644. https://doi.org/10.4161/worm.25644

17. Scheufler C, Brinker A, Bourenkov G, Pegoraro S, Moroder L, Bartunik H, Hart FU, Moarefi I (2000) Structure of TPR domain-peptide complexes: critical elements in the assembly of the Hsp70-Hsp90 multichaperone machine. Cell 101:199-210. https://doi.org/10.1016/s0092-8674(00)80830-2

18. Scoto M, Cullup T, Cirak S, Yau S, Manzur AY, Feng L, Jacques TS, Anderson G, Abbs S, Sewry C, Jungbluth H, Muntoni F (2013) Nebulin (NEB) mutations in a childhood onset distal myopathy with rods and cores uncovered by next generation sequencing. Eur J Hum Genet 21:1249-1252. https://doi. org/10.1038/ejhg.2013.31

19. Srikakulam R, Liu L, Winkelmann DA (2008) Unc45b forms a cytosolic complex with Hsp90 and targets the unfolded myosin motor domain. PLoS One 3:e2137. https://doi.org/10.1371/journal.pone.0002137

20. Wang H, Schanzer A, Kampschulte B, Daimaguler HS, Logeswaran T, Schlierbach H, Petzinger J, Ehrhardt H, Hahn A, Cirak S (2018) A novel SPEG mutation causes non-compaction cardiomyopathy and neuropathy in a floppy infant with centronuclear myopathy. Acta Neuropathol Commun 6: 83. https://doi.org/10.1186/s40478-018-0589-y

21. Wohlgemuth SL, Crawford BD, Pilgrim DB (2007) The myosin co-chaperone UNC-45 is required for skeletal and cardiac muscle function in zebrafish. Dev Biol 303:483-492. https://doi.org/10.1016/j.ydbio.2006.11.027

\section{Publisher's Note}

Springer Nature remains neutral with regard to jurisdictional claims in published maps and institutional affiliations.

Ready to submit your research? Choose BMC and benefit from:

- fast, convenient online submission

- thorough peer review by experienced researchers in your field

- rapid publication on acceptance

- support for research data, including large and complex data types

- gold Open Access which fosters wider collaboration and increased citations

- maximum visibility for your research: over $100 \mathrm{M}$ website views per year

At $\mathrm{BMC}$, research is always in progress.

Learn more biomedcentral.com/submissions 